\title{
Deep Inferior Epigastric Flap Combined Bilateral-Anterolateral Thigh Flap for the Coverage of Both Lower Extremity Soft-Tissue Defect: A Case Report and a Literature Review
}

This article was published in the following Dove Press journal:

Clinical, Cosmetic and Investigational Dermatology

\section{Xilin Liu (D)* \\ Da Huo* \\ Guangzhi Wu}

Department of Hand Surgery, ChinaJapan Union Hospital of Jilin University, Changchun, I30033, People's Republic of China

*These authors contributed equally to this work
Correspondence: Guangzhi Wu

Tel +86-13944I8I019

Email wuguangzhi@jlu.edu.cn
Background/Purpose: Anterolateral thigh perforator (ALTP) flap and deep inferior epigastric perforator (DIEP) flap have been advantageous over traditional myocutaneous flaps as they preserve the integrity of donor site muscles and minimize the damage and complication to donor site structures. Here, we reported the efficacy of free ALTP and DIEP in the repair of large skin area and soft tissue defects on both lower limbs after trauma.

Case Report: A 19-year-old female traffic accident victim presented with multiple open bilateral fractures to the lower extremities with joint dislocation, massive skin and soft tissue defects, and multiple soft tissue contusion. Wounds on both lower extremities were covered with VSD (Vacuum Sealing Drainage). The tibiofibular and ankle joints on both lower limbs were fixed using external fixators. DIEP and ALTP flap were performed at different times.

Results: After transfer, the vascular pedicle was anastomosed to the anterior tibial artery, posterior tibial artery, and the accompanying vein. After the operation, the donor site was directly closed and sutured. All flaps survived with a good appearance, leaving only a linear scar at the donor site.

Conclusion: This case shows that free ALTP and DIEP flaps are ideal for repairing large skin area and soft tissue defects in bilateral lower limbs after trauma.

Keywords: anterolateral thigh perforator flap, deep inferior epigastric perforator flap, soft tissue repair, skin repair

\section{Introduction}

Apart from severe bone infection, a large area of tissue defect after severe lower limb trauma often exposes tendons and bones. Reconstructive surgery may use pedicles (free muscle-skin flaps) or skin grafting. ${ }^{1-4}$ The area of soft tissue defect in the skin of bilateral lower limbs is often large. To achieve limited incision, effective coverage, and restore lower limbs' shape in the recipient area, restore function, and minimize donor site injury, effective free flaps should be selected, such as greater omentum transplantation combined with a free skin graft, lobulated skin flap, or conjoined skin flap, ${ }^{5-7}$ as they can effectively repair most wounds.

Being a reconstructive technique, microsurgical anastomoses is based on raising tissues from healthy body parts with dispensability or excess, in advance to transplant them to the areas where they lack, mainly after chronic infection, oncological surgery, or trauma. A microsurgical transfer from a strict point of 
view implies a double vascular anastomosis less than $3 \mathrm{~mm}$ between vessels in the transferred tissue to the ones in the recipient area. ${ }^{8}$ Right from their inception, reconstructive techniques have been widely implied for restoring the form, function, and integrity of the body. ${ }^{9}$ Though plastic surgery is related to medicine discipline for bringing all these techniques together, however, it lacks an anatomical limitation; thus, its knowledge is widespread according to the diverse body regions through ophthalmology, maxillofacial surgery, hand surgery, etc.

However, problems like skin graft contracture and limited repair area are still challenging for the surgeons. Here, we present a case report of a successful three-staged repair of a large soft-tissue defect of lower limbs using bilateral anterolateral thigh perforator (ALTP) flap combined with deep inferior epigastric perforator (DIEP) flap.

\section{Case Report}

A 19-year-old female traffic accident victim was presented with multiple open bilateral fractures to the lower extremities with joint dislocation, massive skin and soft tissue defects, and multiple soft tissue contusion. Wounds on both lower extremities were covered with VSD (Vacuum Sealing Drainage). The tibiofibular and ankle joints on both lower limbs were fixed using external fixators. Knee joints' passive motion on lower limbs was normal. The active movement was limited to a motion range of about 20 degrees due to pain. Muscle force was grade IV. Ten days later, wound exudation of both lower limbs decreased, granulation tissue grew fresh, and large areas of tendon, bone, and joint got exposed. Because of mandible fracture reduction and internal fixation, tracheotomy and indwelling tracheal cannula and artificial nose lead to the inability of spontaneous sputum excretion, and the condition requires long-term bed rest. Moreover, the operation time of DIEP combined with ALTP flap is significantly prolonged, which will definitely increase the risk of postoperative pulmonary complications. After comprehensive consideration, the operation was performed at different times. The skin on the right leg had a circular defect and the tibia was exposed. An anterolateral thigh flap $(16 \mathrm{~cm} \times 7 \mathrm{~cm})$ was designed from the opposite thigh for covering the tibia and soft tissue defects of the right leg (Figure 1). The vascular pedicle was anastomosed to the anterior tibial artery, and the donor site was directly sutured. Cutaneous deficiency on the right leg was repaired by skin grafting from the left thigh.

Ten days later, the blood supply to the skin flap and skin graft area on the right lower limb was good, most of the 2 nd toe on the left foot was necrotic, palmar skin defect was observed on the 1st metatarsal joint, and the joint capsule was exposed. The 3rd metatarsal bone had dry necrosis, without surrounding soft tissue. The necrotic tissues were removed. After bone cement removal from the left leg, the defect area of the skin and soft tissue was about $23 \mathrm{~cm} \times 10 \mathrm{~cm}$. The fibular artery appeared to be of good texture. However, the anterior tibial artery was located at the proximal tibial stump, with poor texture. A skin flap of the same size was designed on the abdomen (Figure 2) and sutured to the skin defect area of the left leg. The trunk of the right inferior epigastric artery was anastomosed to the internal pressurized blood vessel reserved at the left pedicle (Figure 2). The left inferior epigastric artery was anastomosed to the peroneal artery and the vein to the peroneal vein. Seven days later, an anterolateral thigh flap was designed on the right thigh to cover the wound of the left dorsum of the foot combined with a skin graft (Figure 3). The branch of the posterior tibial artery was anastomosed to the descending branch of the lateral circumflex femoral artery of the flap. The vein
A

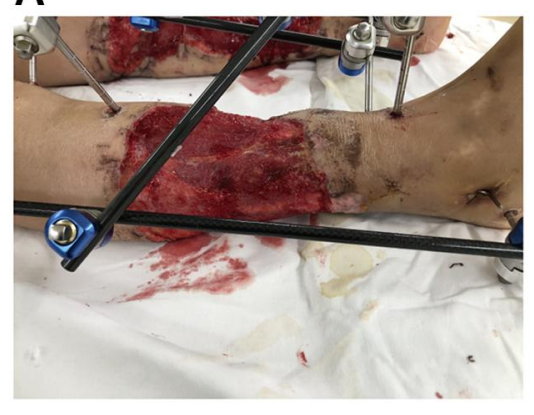

B

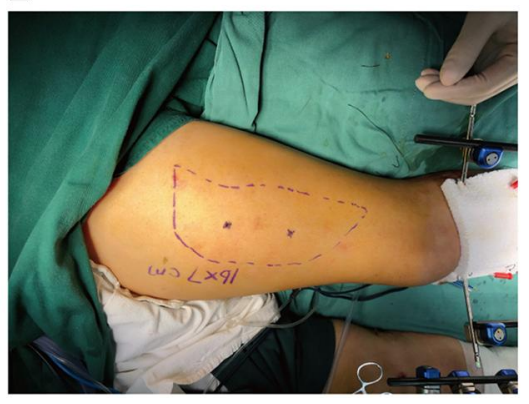

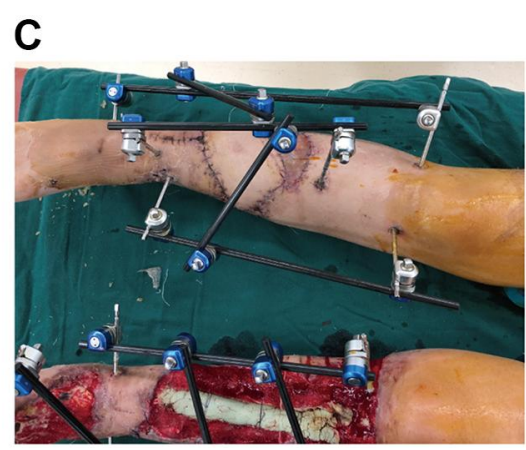

Figure I Repair of the right leg. (A) Preoperative view of the recipient site (right lower limb). (B) ALTP flap was designed to cover the soft-tissue defect. (C) Postoperative view of the recipient site at 2 weeks follow-up. 
A

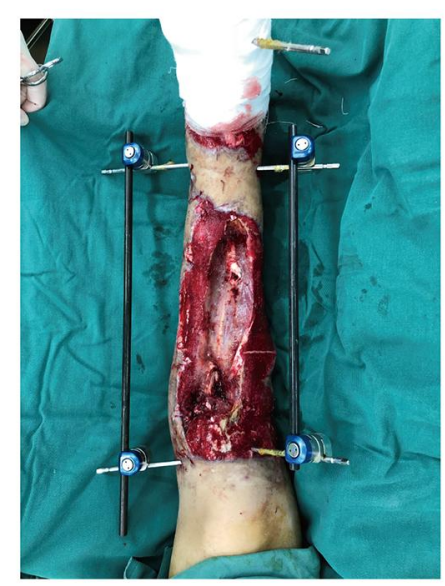

C

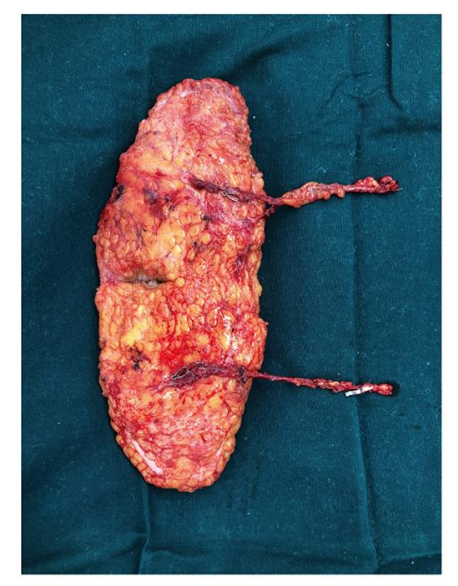

D

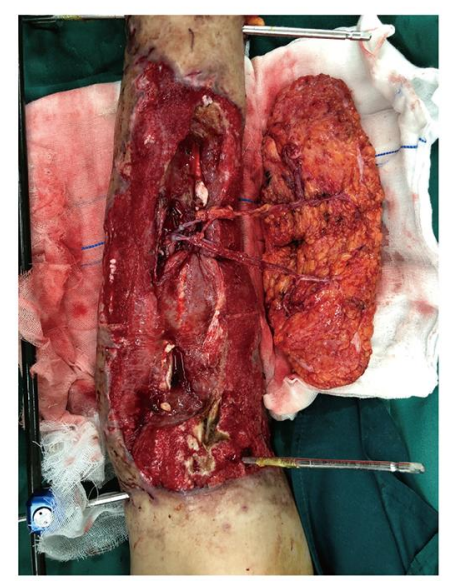

B
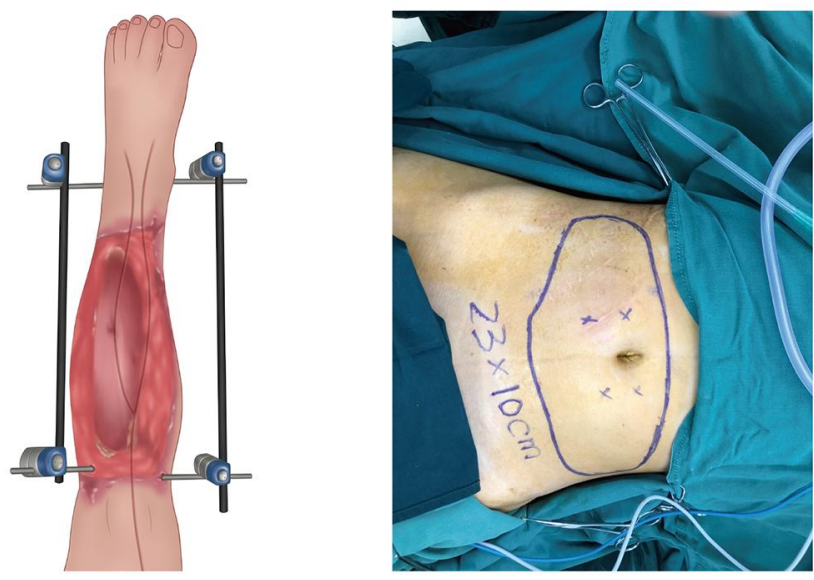
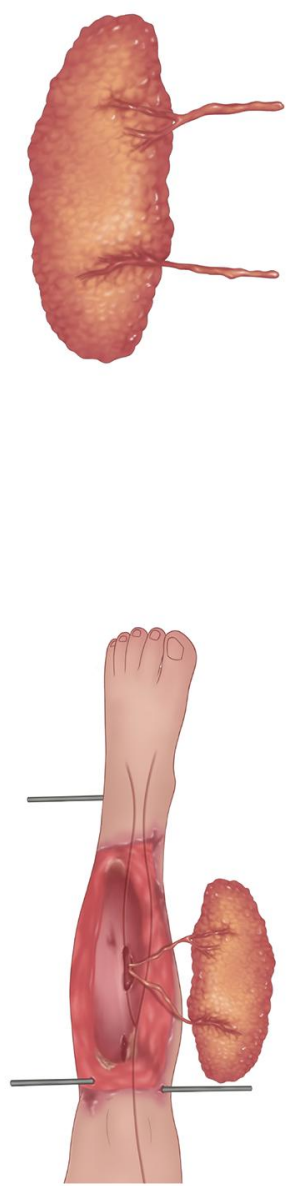

Figure 2 Repair of the left leg. (A) Preoperative view of the recipient site (left lower limb). (B) Skin marking for the DIEP flap with perforators marked using a hand-held Doppler probe. (C) DIEP flap was elevated to reconstruct the very large soft-tissue defect. (D) A DIEP flap was harvested as microanastomoses performed to the peroneal artery and vein. 
A

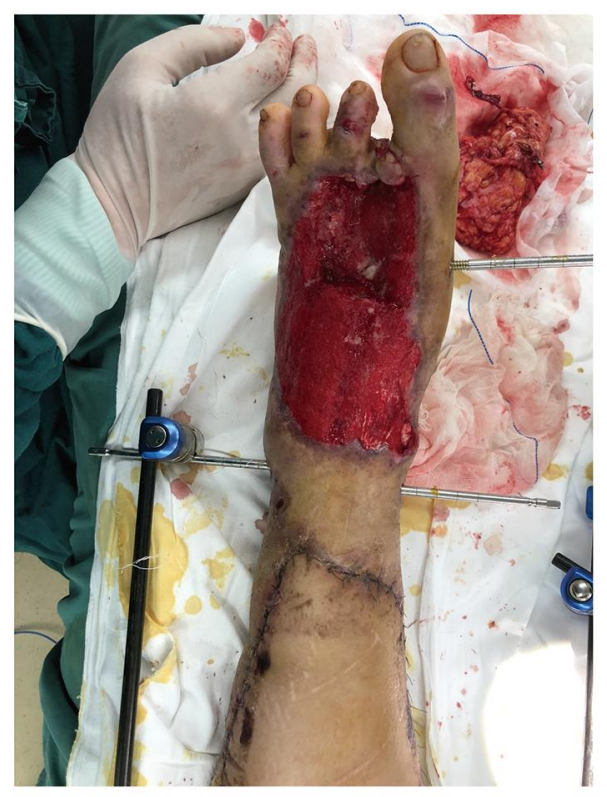

C

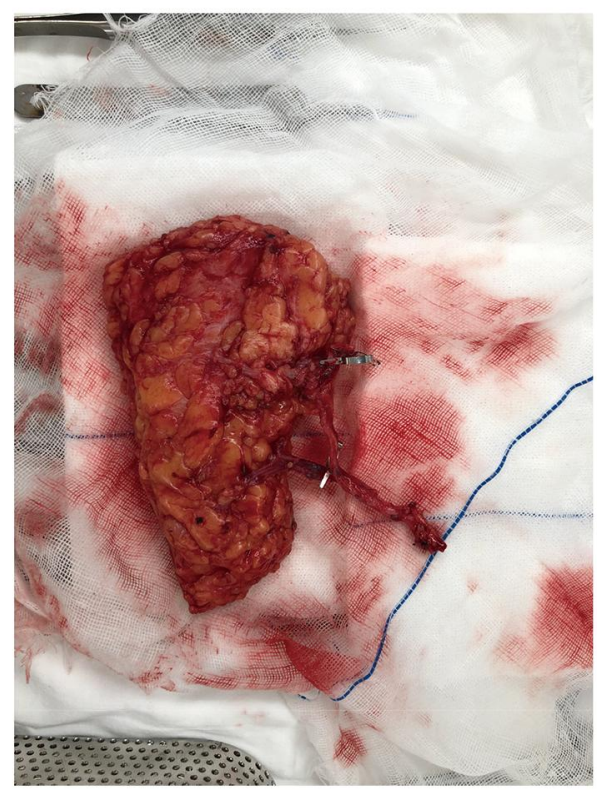

B

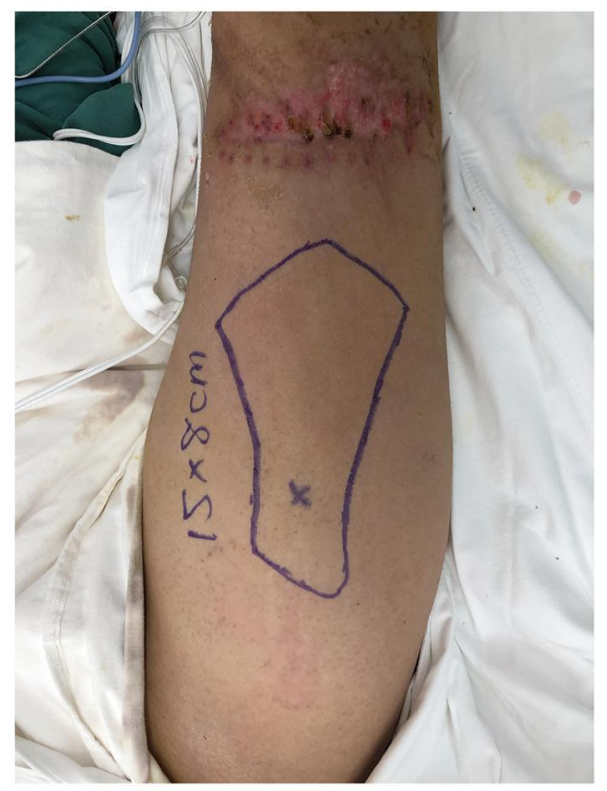

D

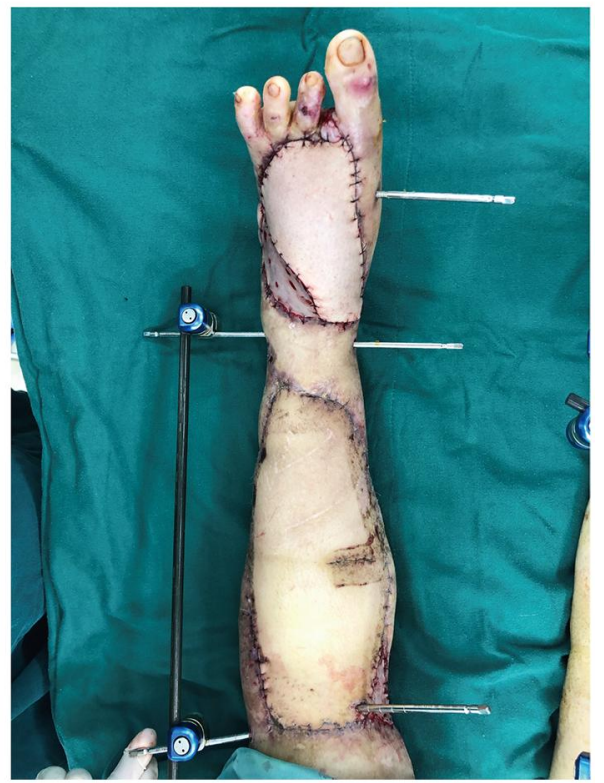

Figure 3 Repair of the left foot. (A) Preoperative view of the recipient site. (B) An anterolateral thigh flap supplied by a midthigh. (C) The anterolateral thigh flap was elevated and can be transposed directly onto the large defect. (D) Note the tension free closure of the defect, the good color and texture matching.

was anastomosed to the vein of the descending branch of the lateral circumflex femoral artery with the posterior tibial artery. The blood supply to the flap was good. All flaps were sutured to the skin edge at the recipient site without tension. All donor sites were closed directly in one stage. After 9 months of follow-up, the flaps survived well, with a satisfactory appearance. Donor sites on both thighs and abdomen healed well. The lower limbs were saved successfully (Figure 4).

\section{Discussion}

Mechanical injury, such as in traffic accidents, often causes serious tissue damage, extensive muscle necrosis, and severe injury to bones, blood vessels, nerves, and tendons. Lower limb injury is common in the lower limb skin and soft tissue defects such as tibia, fibula, and ankle joint. Most of these are accompanied by bone and joint exposure. Lower limb wound reconstruction is challenging. In repairing skin and soft tissue defects in lower 


\section{A}

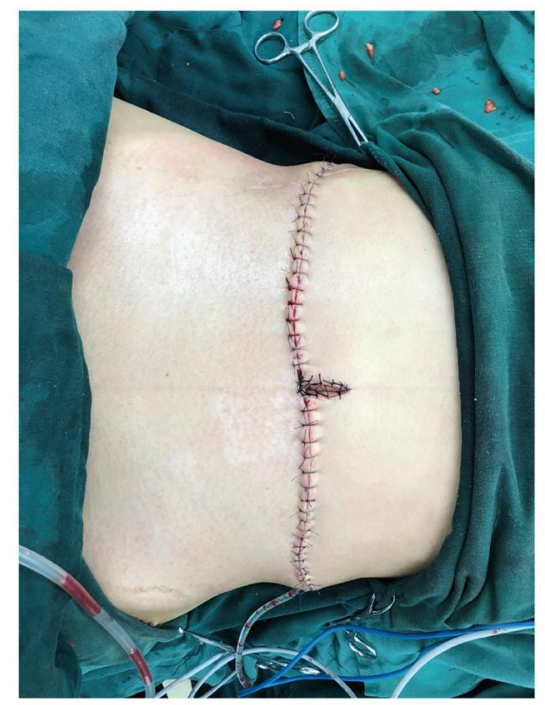

C

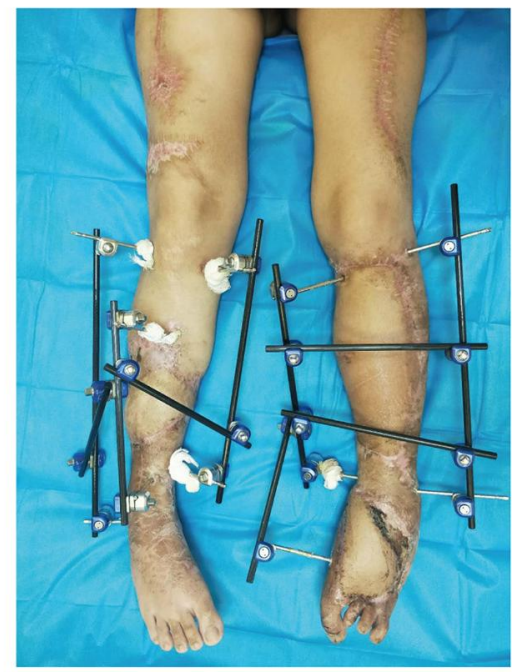

B
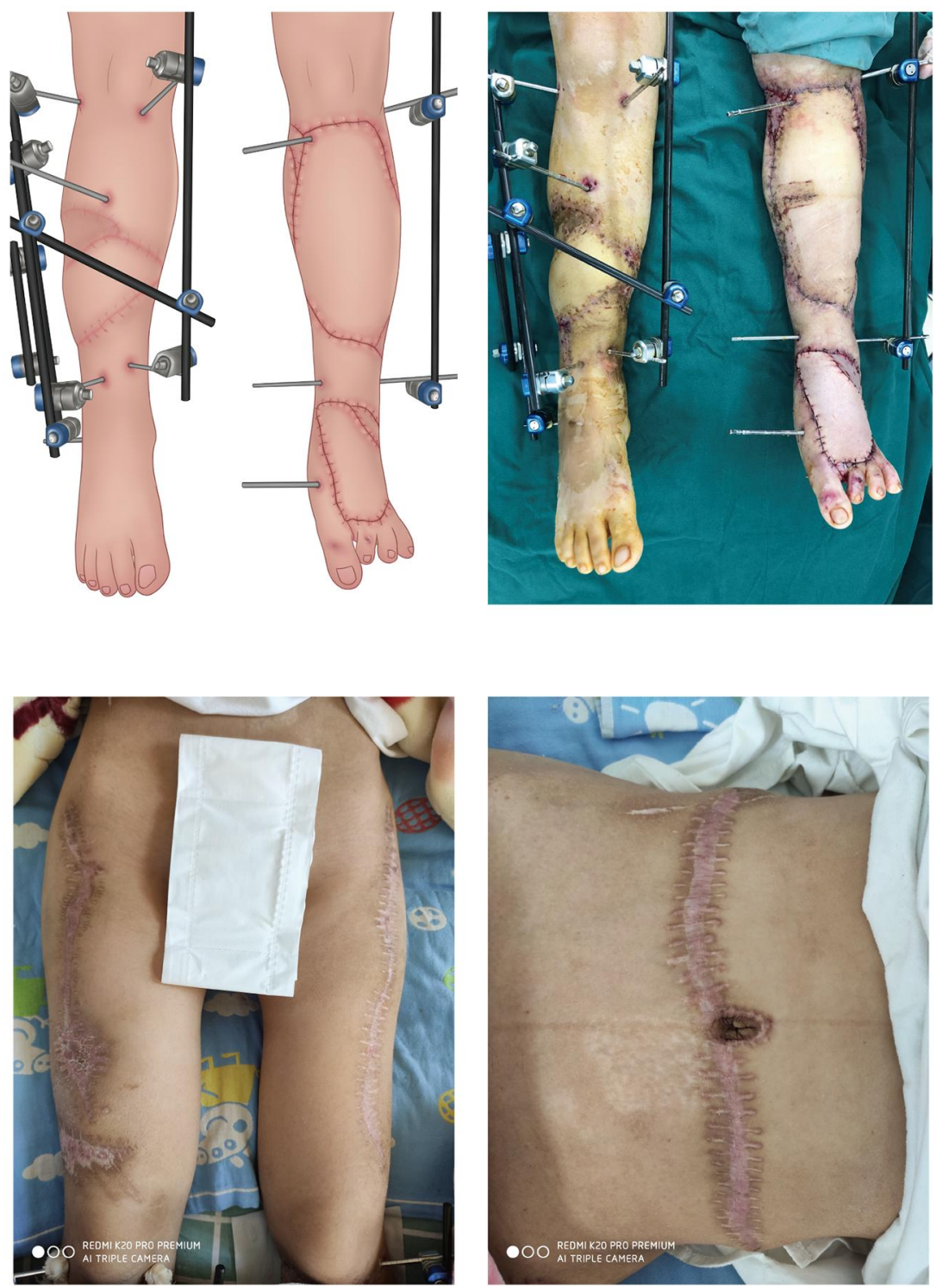

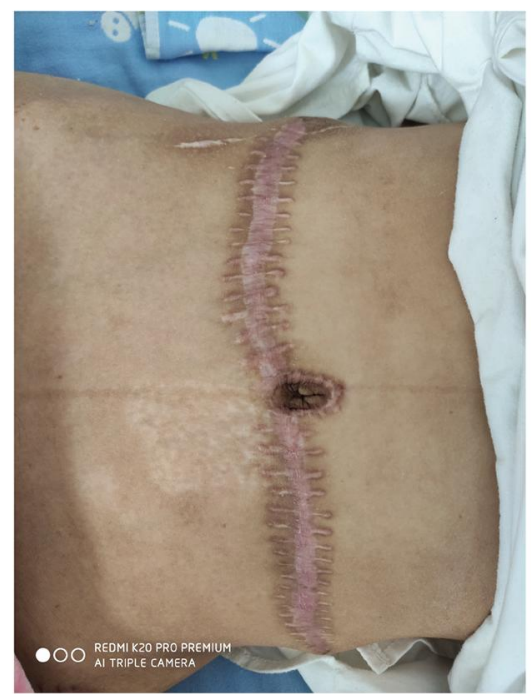

Figure 4 Postoperative follow-up. (A) All donor sites were closed directly in one stage. (B) Follow-up at 2 months view. (C) Follow-up at 9 months view.

limbs, the effective method is that which can improve and optimize foot function, reduce adhesion, and cover the bone. Based on the wound site, the extent of the injury, and the peripheral blood vessel situation, appropriate flaps should be considered to cover the wound completely. Flap thickness should be appropriate, and it should be able to reconstruct sensation. After thorough debridement and stable internal fixation, choosing a skin flap with similar color and thickness, sensation, appearance, having less impact on donor site, and with no need for secondary plastic surgery to cover the wound surface, is challenging.
Before the development of free graft repair, there were few methods to reconstruct lower limb wounds, especially for the distal one-third of the leg. With rapid surgical advancements, the free flap has been widely used. For defects in lower extremities, there is a paucity of local blood supply and soft tissue, thus making the process highly challenging for surgeons. The skin grafting, direct closure, and local flaps including cross-leg flap, muscle flap, and free flap are among the methods used for reconstruction of the defects in lower extremities. The use of fasciocutaneous, musculocutaneous, and cutaneous flaps along specific dominant 
vascular pedicles have been employed for the direct transfer of tissues that are show less dependency on the wound bed blood supply. They have also been found effective for introducing new circulation to the surface of the leg and present a longer and reliable wound coverage option. ${ }^{10}$ Recently, there has been significant interest in perforator flaps, which have significantly improved lower limb wound reconstruction. ${ }^{1}$ The pedicled myocutaneous flap has been used as a major method of repairing skin and soft tissue defects in lower limbs. This type of skin flap has clear major blood vessels and a reliable, safe blood supply and can cover a large area. Muscle looseness after myocutaneous flap transplantation affects the function and aesthetics of wound repair, which often occurs at various degrees. ${ }^{2}$ The concept of perforator flap was first proposed by Koshima and Soeda in $1989 .^{3}$ This is an island skin flap supported by a perforator artery and vein and belongs to the axial vascular flap. In 2003, Halleck pointed out that perforator flap refers to the flap supported by perforators like intermuscular septal perforator and muscular perforator. Perforator flaps may or may not contain deep fascia. ${ }^{4}$ Additionally, this kind of flap causes less damage to the donor site and leaves a beautiful appearance after operation.

This case reported in the present study was treated by selective operation. The wound surface of the recipient area should undergo complete debridement before skin flap transplantation. The edge of the wound was excised by $0.5-$ $1.0 \mathrm{~cm}$. Inflammatory granulation tissue was completely removed. Granulation and wound secretion cultures were negative for bacteria and fungi. Preoperative x-ray and MRI examination revealed no medullary cavity infection in the exposed bone, and the exposed bone cortex was removed by $3-5 \mathrm{~mm}$. Skin flap design aimed to detect the artery perforating point. At least 2 groups of vascular perforating points in different parts of the flap were obtained before the operation. Doppler was used to detect and mark the perforating point of perforator vessels near the midpoint of the flap axis connecting the anterior superior iliac spine to the outer and upper edge of the patella. Based on defect area and wound shape, a suitable skin flap area and shape were bilaterally designed in the anterolateral thigh region. At least 2 groups of vascular perforating points in different parts of the flap were obtained before the operation. Doppler was used to detect and mark the perforating point of perforator vessels near the midpoint of the flap axis connecting the anterior superior iliac spine to the outer and upper edge of the patella. When cutting the anterolateral thigh flap, the lateral edge of the flap is cut first. Skin perforator vessels on the descending branch of the lateral circumflex femoral artery passing through the fascia lata are then carefully located. All perforators found were completely preserved to determine if there were perforators in the flap. If the flap perforator's position differs from that of the preoperative design, the flap position should be adjusted in time. Based on the position line of the reserved perforator, the fascia lata was incised longitudinally. Neuromuscular branches accompanying perforator vessels should be kept as much as possible to reduce nerve injury. The flap should be thinned before its blood supply is cut off. Attention should be paid to the distribution of thick blood vessels in the thinning plane to avoid damage. The descending branch of the lateral circumflex femoral artery and its accompanying veins were used as anastomotic vessels. To increase flap pedicle length, the flap can be designed upward and the blood vessel perforating point should be included in the proximal end of the flap. The perforator should be carefully operated from the rectus abdominis muscle to prevent traction injury. When dissecting perforator vessels from the rectus abdominis, the cross-section of muscle fibers should be reduced as much as possible to minimize damage to the rectus abdominis. If the medial or the lateral branch is cut, the nerve entering the rectus abdominis from its lateral edge should be avoided to avoid denervation. Because this case involved a young woman, to retain beauty as much as possible, the navel was preserved.

\section{Characteristics of Lower Limbs Skin Defects}

Skin and soft tissue defects after lower limb trauma are relatively serious. In most patients, tendon, joint, nerve, and blood vessel exposure, easily causes wound dehiscence, chronic infection, and even dysfunction, which complicate treatment and rehabilitation. Thus, it is critical to select a suitable donor site for skin and soft tissue repair after lower extremity trauma. Foot and ankle soft tissue injury is usually caused by the high-energy impact that severely crushes soft tissue and damages blood supply. This is often accompanied by serious trauma, including fracture, joint dislocation, and nerve injury. Subcutaneous fat in the sole has tight tissue, and the subcutaneous ligament is closely connected to plantar aponeurosis and the fibrous cord. Deep tissue is often only slightly damaged. However, the subcutaneous 
ligament on the back of the foot is loose, which is more likely to cause soft tissue injury or tear. The soft tissue avulsion injury on the back of the foot can be avulsed from the superficial layer of the deep fascia. The plantar side is mostly from the superficial layer of the plantar aponeurosis. While superficial veins of the avulsed skin are injured, arteries are generally retained in the proximal end. There are no blood vessels in the avulsed skin, which greatly complicates follow-up treatment. Severe leg trauma may cause extensive skin and soft tissue defect and bone exposure. Thus, free tissue transplantation should be chosen to repair such wounds. When the defect area of skin and soft tissue in the functional part of the foot is large, the pedicled flap with a neurovascular bundle or free flap should be chosen for repair while retaining the texture, thickness, and mobility of the donor site.

\section{Advantages and Disadvantages of Different Flaps}

Advancements in microsurgical techniques have increased the types of flaps in clinical use. Perforator flaps like ALTP, DIEP, and TAPF have unique advantages in repairing largearea soft tissue defects in limbs. ${ }^{11}$ Additionally, the flaps have a good texture, wear resistance, and good elasticity, which promotes recovery of foot function after the operation.

(1) Use of pedicled flaps to repair soft-tissue defect in lower limbs is relatively simple, with no need to anastomose blood vessels. However, there is a chance of necrosis in the distal part of the flap, and its pedicle may appear bloated, while the area and repair site of the flap is limited. The anterolateral thigh pedicled skin flap can repair knee joint and upper leg defects, but it is not suitable to use a pedicled skin flap when the limb is serious damage or injury below the knee. There is serious injury around the wound surface of the ipsilateral limb.

(2) The free flap can be used to repair soft tissue defects on lower limbs. The flap with a large area can be cut, and the affected area is not limited. It is suited for repairing large area soft-tissue defects but requires high microsurgical technology. Perforator flaps also include axial blood vessels and can effectively resist infection and occlude dead space in the treatment of implanted human infection and osteomyelitis. It has the same advantages as musculocutaneous flap. ${ }^{7}$

\section{Anterolateral Thigh Flap}

The anterolateral thigh flap is widely used. The length of the vascular pedicle is usually $>8 \mathrm{~cm}$ while the diameter of the blood vessel is about $2 \mathrm{~mm}$, making the flap easier to operate. ${ }^{12}$ Free anterolateral thigh flap can repair different types of lower extremity wounds, ${ }^{13,14}$ including covering heel and dorsum skin wounds. The flap can carry anterolateral femoral cutaneous nerves to reconstruct skin flap sensation. It can also carry fascia lata, lateral femoral muscle, or rectus femoris as composite flaps. The perforator vessel anatomy of the flap was constant. There are $(9.5 \pm 4.5)$ perforating branches from the lateral circumflex femoral artery system into the anterolateral femoral region. The ascending, transverse, and descending branches of the perforator branches are all connected to the skin. In the anterolateral thigh perforator flap, the maximum area of a single perforator flap was $35 \mathrm{~cm} \times 25 \mathrm{~cm} .{ }^{11}$ In our case, the unilateral flap was $16 \mathrm{~cm} \times 7 \mathrm{~cm}$ (Figure 1). The donor site was closed directly, leaving only a linear scar and the flap.

The lateral circumflex femoral artery mainly originates from the deep femoral artery but it may also originate from the femoral artery. There are ascending, transverse, and descending branches. The vascular pedicle is long, and the branches are thick. Past studies show that the internal diameters of the ascending, transverse, and descending branches are $(2.55 \pm 0.25),(2.43 \pm 0.35)$, and $(3.50 \pm 0.34)$, respectively. ${ }^{15}$ In this case, the descending branch of the anterolateral femoral artery was anastomosed with the anterior tibial artery, and blood flow to the reconstruction flap was stable. There are abundant perforator vessels and tissues in the anterolateral thigh region. The skin flap can be used to repair the lower limb superficial wounds via microdissection. The chimeric muscle flap was used to repair the wound with a deep dead cavity, achieving 3-dimensional restoration of the receiving area. The flap can carry the lateral femoral cutaneous nerve or branch and suture with the peripheral cutaneous nerve stump to restore sensation. It is suitable for repairing wounds with high demand for hand or foot sensory reconstruction. Here, we used the lateral femoral cutaneous nerve to reconstruct flap sensation.

\section{Deep Inferior Epigastric Perforator Flap}

Koshima et al were the first to describe deep inferior epigastric perforator flap (DIEP). It is thought that the main nutrient vessel for the flap is the inferior epigastric artery and the perforator vessel is constant. ${ }^{3}$ Taylor et $\mathrm{al}^{16}$ found that the blood supply to the skin in the anterolateral abdominal wall was multi-source and the blood supply of DIEP was classified, 
providing clinicians with a better understanding of the musculocutaneous perforator of the inferior epigastric artery. The DIEP flap has become one of the most widely used perforator flaps. Based on the vascular pedicle, it can be divided into a single pedicle chimeric flap, double pedicle flap, multipedicle flap, or a union flap spanning the anatomic area. However, for patients with a median incision in the lower abdomen, or with a flat lower abdomen, the flap with a single vascular pedicle cannot provide enough tissue for large-area tissue repair. Double pedicled flaps can provide blood supply for larger tissues but they need blood supply reconstruction for the 2 vascular pedicles during transplantation. The DIEP flap has the advantages of a transverse rectus abdominis musculocutaneous flap (TRAM) flap, including adequate tissue and blood supply. The donor site is hidden, and the tissue is loose. The rectus abdominis and anterior sheath are reserved. The donor site is less damaged.

Most flaps can be closed and sutured directly after cutting, without additional donor wounds. The postoperative recovery time is short and meets aesthetic requirements. Most patients do not need to change their position. Donor and recipient site operations can be carried out at the same time, shortening operation time. Vessel diameter at the donor site of the abdominal wall and those in the recipient area of the lower extremities, including the anterior and posterior tibial vessels, are more suitable for vascular anastomosis. In our case, it was to increase pressure difference between the artery and vein in the perforator flap, reduce blood flow resistance in the flap, and ensure unobstructed blood flow to all parts of the perforator flap, thereby enhancing the survival of the large flap. During the operation, the DIEP flap was designed with an inferior epigastric artery as the axial vessel. The transverse incision was consistent with the abdominal skin texture direction, and the position was hidden. The donor site was directly closed and sutured, leaving a linear scar only. Because the patient's condition was serious, the operation was performed at different times. If the patient's whole body is in good condition and there are no multiple injuries, the combined flap is also a good choice.

Primary closure of the donor site can be achieved with a linear scar. Although the terminal branches of the 10th to 11th intercostal nerves can be anastomosed, the DIEP flap lacks a single innervation. Additionally, the area of nerve donor skin was small, and the nerve pedicle is often short and scattered. The skin flap may have poor sensory recovery, wear-resistance, and local chronic ulceration. Thus, it is not suited for repairing palms, soles, and other parts with high sensory requirements. Clinicians should be careful when confirming the indication of the skin flap. It takes a long time to dissect the vascular pedicle of the rectus abdominis. Moreover, the anatomy of the inferior epigastric artery and its perforator may vary. Therefore, it is necessary to detect the inferior abdominal wall vessels with color Doppler before the operation and to explore the position, caliber, and blood flow of perforating vessels. These will provide an accurate reference for marking the abdominal skin flap and enhance the operation's success.

Furthermore, the limb salvage therapy used for soft-tissue sarcoma involves post- or preoperative administration, surgery associated with radiotherapy (RT), histological type dependency, margins of surgical resection, and the grade of differentiation. ${ }^{16}$ Described by Allen and Treece for the first time, the deep inferior epigastric perforator (DIEP) flap, is a reliable and versatile voluminous flap commonly employed for the reconstruction of the breast, can also be a promising alternative for the coverage of large soft-tissue defects in other body parts. ${ }^{17}$ The transfer of free tissue for the defects of calvarial and scalp after infection, trauma, or tumor extirpation has been reported for achieving satisfactory results. Further, the advantages of the anterolateral thigh flap include its harvesting as myocutaneous, fasciocutaneous, and adipofascial flap based on the requirements of the defect. The fascia can be employed for duraplasty in skull-based defects. Similarly, the myocutaneous design can be advantageous in case dead space is present. ${ }^{18,19}$

In conclusion, the use of ALTP and DIEP flap to repair large-area soft tissue defects in lower limbs has good clinical effects, flexible design, satisfactory recipient area shape, and direct donor site closure, which leaves an only linear scar. These factors can effectively improve limb motor function, highlighting the technique's high clinical application value.

\section{Ethics Statement}

Appropriate written informed consent was obtained from the patient, and she agreed to have the case details and any accompanying images published.

\section{Disclosure}

The authors report no conflicts of interest in this work.

\section{References}

1. Iorio ML, Cheerharan M, Olding M. A systematic review and pooled analysis of peroneal artery perforators for fibula osteocutaneous and perforator flaps. Plast Reconstr Surg. 2012;130(3):600-607. doi:10.1097/PRS.0b013e31825dbff3 
2. Sameem M, Au M, Wood T, Farrokhyar F, Mahoney J. A systematic review of complication and recurrence rates of musculocutaneous, fasciocutaneous, and perforator-based flaps for treatment of pressure sores. Plast Reconstr Surg. 2012;130(1):67e-77e. doi:10.1097/ PRS.0b013e318254b19f

3. Koshima I, Soeda S. Inferior epigastric artery skin flaps without rectus abdominis muscle. Br J Plast Surg. 1989;42(6):645-648. doi:10.1016/0007-1226(89)90075-1

4. Hallock GG. The medial circumflex femoral (gracilis) local perforator flap-a local medial groin perforator flap. Ann Plast Surg. 2003;51 (5):460-464. doi:10.1097/01.SAP.0000096149.92205.44

5. Bota O, Spindler N, Sauber J, Aydogan E, Langer S. Double-pedicled free deep inferior epigastric perforator flap for the coverage of thigh soft-tissue defect. Plastic and reconstructive surgery. Global Open. 2017;5(8):e1372.

6. Wong $\mathrm{CH}$, Wei FC, Fu B, Chen YA, Lin JY. Alternative vascular pedicle of the anterolateral thigh flap: the oblique branch of the lateral circumflex femoral artery. Plast Reconstr Surg. 2009;123 (2):571-577. doi:10.1097/PRS.0b013e318195658f

7. Yan H, Yang M, Lineaweaver WC, Myers RS, Chen H, Zhang F. Flap reconstruction of distal lower extremity wounds in diabetic patients. Ann Plast Surg. 2011;67(4):426-428. doi:10.1097/SAP.0b013e 3181 fab99e

8. Masia J, Olivares L, Koshima I, et al. Barcelona consensus on supermicrosurgery. $J$ Reconstr Microsurg. 2014;30(1):53-58. doi:10.1055/s-0033-1354742

9. Gulgonen A, Gudemez E. Toe-to-hand transfers: more than 20 years follow-up of five post-traumatic cases. J Hand Surg Br. 2006;31 (1):2-8. doi:10.1016/J.JHSB.2005.09.017

10. Wells MD, Bowen CV, Manktelow RT, Graham J, Boyd JB. Lower extremity free flaps: a review. Can J Surg. 1996;39(3):233-239.
11. Koshima I. Free anterolateral thigh flap for reconstruction of head and neck defects following cancer ablation. Plast Reconstr Surg. 2000;105(7):2358-2360. doi:10.1097/00006534-200006000-00007

12. Lakhiani C, Lee MR, Saint-Cyr M. Vascular anatomy of the anterolateral thigh flap: a systematic review. Plast Reconstr Surg. 2012;130(6):1254-1268. doi:10.1097/PRS.0b013e31826d1662

13. Lee MJ, Yun IS, Rah DK, Lee WJ. Lower extremity reconstruction using vastus lateralis myocutaneous flap versus anterolateral thigh fasciocutaneous flap. Arch Plast Surg. 2012;39(4):367-375. doi:10.5999/aps.2012.39.4.367

14. Wong CH, Goh T, Tan BK, Ong YS. The anterolateral thigh perforator flap for reconstruction of knee defects. Ann Plast Surg. 2013;70 (3):337-342. doi:10.1097/SAP.0b013e318234e8e2

15. He J, Tang J, Qing L, et al. A comparative study of selective CT arteriography versus CT angiography on three-dimensional display of perforators in anterolateral thigh perforator flap. Chin J Clin Anat. 2017;35(5):508-512.

16. Taylor GI, Palmer JH. Angiosome theory. Br J Plast Surg. 1992;45 (4):327-328. doi:10.1016/0007-1226(92)90063-4

17. Allen RJ, Treece P. Deep inferior epigastric perforator flap for breast reconstruction. Ann Plast Surg. 1994;32(1):32-38. doi:10.1097/ 00000637-199401000-00007

18. van Driel AA, Mureau MAM, Goldstein DP, et al. Aesthetic and oncologic outcome after microsurgical reconstruction of complex scalp and forehead defects after malignant tumor resection: an algorithm for treatment. Plast Reconstr Surg. 2010;126(2):460-470. doi:10.1097/PRS.0b013e3181de2260

19. Heller F, Hsu CM, Chuang CC, Wei KC, Wei FC. Anterolateral thigh fasciocutaneous flap for simultaneous reconstruction of refractory scalp and dural defects. Report of two cases. J Neurosurg. 2004;100(6):1094-1097. doi:10.3171/jns.2004.100.6.1094

\section{Publish your work in this journal}

Clinical, Cosmetic and Investigational Dermatology is an international, peer-reviewed, open access, online journal that focuses on the latest clinical and experimental research in all aspects of skin disease and cosmetic interventions. This journal is indexed on CAS.
The manuscript management system is completely online and includes a very quick and fair peer-review system, which is all easy to use. Visit http://www.dovepress.com/testimonials.php to read real quotes from published authors. 